\title{
Environmental Constraints on the Mechanics of Crawling and Burrowing Using Hydrostatic Skeletons
}

\author{
K.M. Dorgan
}

Received: 11 June 2009/Accepted: 2 August 2010/Published online: 19 August 2010

(C) The Author(s) 2010. This article is published with open access at Springerlink.com

\begin{abstract}
Mechanics, kinematics, and energetics of crawling and burrowing by limbless organisms using hydrostatic skeletons depend on the medium and mode in which the organism is moving. Whether the animal is moving over or through a solid has long been considered important enough to distinguish crawling and burrowing as different terms, and in fact the mechanics are very different. Crawlers use mechanisms to increase friction to generate thrust while reducing resistive friction. Burrowers in elastic muds extend their burrows by fracture, whereas sands are fluidized by burrowers much larger than grain sizes and smaller burrowers displace individual grains. Gravitational forces depend on how closely the density of the organism matches that of its fluid surroundings, therefore frictional forces depend on whether the organism is moving through air or water and fluidization on whether sands are saturated or unsaturated.
\end{abstract}

Keywords Burrowing $\cdot$ Crawling $\cdot$ Locomotion .

Biomechanics $\cdot$ Hydrostatic skeleton

\section{Introduction}

Many soft-bodied animals use hydrostatic skeletons to swim, crawl, and burrow, and often use similar movements for these different modes of locomotion, e.g., earthworms use peristalsis for burrowing and crawling and nereidid polychaetes use undulation for swimming, crawling and burrowing [1]. The mechanics of swimming through a fluid

K.M. Dorgan $(\bowtie)$

University of California Berkeley,

Berkeley, CA, USA

e-mail: kelly.dorgan@berkeley.edu have been clearly distinguished from those of crawling over substrata, but the distinction between crawling and burrowing has been less clear, in part because direct visualization of burrowers is impeded by the opacity of soils and sediments. Perhaps more importantly, the mechanical responses of muds and sands to forces are less well understood than those of fluids, which are governed by the Navier-Stokes equations [2]. Recent advances in understanding of the mechanics of muds [3] and sands [4] and their application to burrowing $[5,6]$ suggest that not only are the mechanics of crawling and burrowing very different, but mechanisms of burrowing in sands and muds reflect the differences in mechanical properties of the two media [7].

Although worms use undulation for both swimming and crawling, differences in frictional forces between a crawling worm and the substratum generate thrust, whereas swimmers use lift and drag forces to propel themselves forward in a fluid medium. Smooth-bodied worms such as nematodes, some polychaetes, and archiannelids swim using posterior-traveling lateral undulatory waves [8], whereas freshwater oligochaetes use helical waves rather than undulation within a plane [9]. Undulatory waves of nereidid polychaetes travel toward the anterior, which is less effective at generating forward thrust with the body. Instead, the large parapodia act as paddles to provide the driving force, and because the undulatory wave travels anteriorly the parapodia behind the ones exerting forces have just completed a stroke and are held against the body out of the way of fluid flow [10]. Crawling and swimming movements of nereidids are similar, although swimming worms have greater amplitudes, wave-lengths, and frequencies of undulatory waves than crawlers [11]. The mechanics and energetics of crawling and swimming have been compared for the nudibranch, Melibe leonina, which uses a lateral bending mechanism, essentially an undulatory wave with a wavelength equal to the body length, to swim 
[12]. Interestingly, although the mechanics of swimming and crawling are very different and the oxygen consumption rates were much higher for swimmers, the velocity of swimming was also much higher, resulting in a comparable cost of transport [13].

Locomotion with a hydrostatic skeleton has been found to be more energetically expensive than legged locomotion but most studies have focused on terrestrial crawling, e.g., by slugs [13], caterpillars [14], and larval diptera [15]. Energetic cost of limbless locomotion by snakes moving by lateral undulation is comparable to the cost of running by similarly sized animals [16], suggesting that the high cost measured for other limbless animals is a disadvantage of using hydrostatic skeletons. Casey [14] attributes the high cost of a caterpillar's crawling to short stride length and suggests that its hydrostatic skeleton is a biomechanical limitation. The high cost of gastropod locomotion largely results from the cost of mucus production, although inefficiencies in crawling with a hydrostatic skeleton are also a factor [13]. The cost of transport for abalone crawling underwater, however, is much lower than that of slugs [17], and studies of burrowing in marine muds $[18,19]$ likely overestimated the cost of transport because the mechanics of burrowing were not understood $[5,7]$. The cost of maintaining body posture against gravitational forces in air may be an important factor distinguishing terrestrial from aquatic hydrostatic locomotion, although direct comparisons are lacking.

Consideration of the mechanical responses of soils and sediments to burrowers is essential in understanding the mechanics of burrowing and in comparing burrowing behaviors to those for crawling and swimming. It has been well established that the mechanics and energetics of running, flying, and swimming depend strongly on the material properties of the environment, including the stiffness of solid substrata and viscosity and density of fluids [1,20]. Surface stiffness affects running efficiency and performance [21], and the energetic cost of walking and running on sand is higher than on compact terrain [22]. Mechanics of swimming and flying depend on fluid viscosity and density, as succinctly encapsulated in the Reynolds number for the resulting flow [2]. This review provides a brief overview of the mechanics of crawling, burrowing in muds and sands, and moving through a tube or permanent burrow, including relevant material properties, forces, and components of external work. Differences in mechanics of these modes of locomotion indicate that crawling and burrowing are distinct even though the behaviors observed are similar.

\section{Locomotion with a Hydrostatic Skeleton}

Hydrostatic skeletons are internally pressurized systems against which antagonistic muscles act to change body shape and to move. Muscular hydrostats have a constant volume, so muscle contraction that shortens the hydrostat in one dimension results in extension in another direction [23]. Caterpillars and worms with open coeloms are one large hydrostat; earthworms and some polychaetes have septa that divide the body into separate segments, each of which maintains constant volume. Many worms also use their hydrostatic skeletons to evert a proboscis or pharynx, which are extensions of the body wall or throat region respectively that are used for feeding and burrowing [24]. Contraction of both circular and longitudinal muscles simultaneously increases body pressure, enabling eversion of the proboscis or pharynx out of the mouth, much like inflating a balloon. Many hydrostats are reinforced with inextensible helical fibers that affect how the shape changes with increased pressure [24, 25].

Different mechanisms of crawling with a hydrostatic skeleton include peristalsis (i.e., many worms), in which circular and longitudinal muscles contract alternately to extend the body then to pull the posterior forward, and undulation (i.e., nematodes, nereidid polychaetes), in which longitudinal muscles on opposite sides of the body contract alternately. Waves of muscular contraction move anteriorly in direct peristalsis, which requires an open body cavity and movement of fluid among segments. In retrograde peristalsis, the segments maintain constant volume and the wave of muscular contraction progresses in the opposite direction of movement [26]. Crawlers also use a muscular foot (i.e., gastropods), proleg appendages (i.e., caterpillars), or anterior and posterior suckers (i.e., leeches).

\section{Crawling on a Surface}

In order to understand how organisms use hydrostatic skeletons to move, it is important to consider how their physical environment affects the type and magnitude of work done by the animal. The simplest case to consider is crawling on a surface [Fig. 1(a)]. To move forward on a surface, part of the body remains in contact with the surface and exerts a backward static frictional force while the rest of the body moves forward, doing work against kinetic friction. At slow speeds, inertial work is not important [1]. Unless the animal is very large or in water in which the external flow is very fast, work against drag is much less important than frictional work.

Alexander [1] describes crawling by peristalsis as a balance between a fraction $q$ of segments moving forward and a fraction $(1-q)$ that remain stationary. The frictional force exerted by stationary segments to move the worm forward, $(1-q) m g \mu_{\text {back}}$, must exceed the frictional force on the moving segments, $q m g \mu_{\text {forward, }}$, for the worm to move 
Fig. 1 Lateral view diagram of worm, shown in gray, crawling on a surface by peristalsis. Nor$\mathrm{mal}(\mathrm{N})$ and frictional forces $\left(\mathrm{Fr}_{\mathrm{s}}\right.$ and $\mathrm{Fr}_{\mathrm{k}}$ for static and kinetic respectively) are indicated for (a) a worm crawling in air, (b) in water, and (c) in water with a mechanism to increase the coefficient of static friction or to grip the substratum. The normal force, $N=\left(\rho_{w}-\rho_{f}\right) V_{s} g$ in which $\rho_{w}$ and $\rho_{f}$ are the densities of the worm and the fluid respectively, $V_{s}$ is the volume of the segment, and $g$ is the gravitational constant. Resulting thrust $(\mathrm{F}=\mathrm{ma})$ is indicated by gray dashed arrows (a) Air

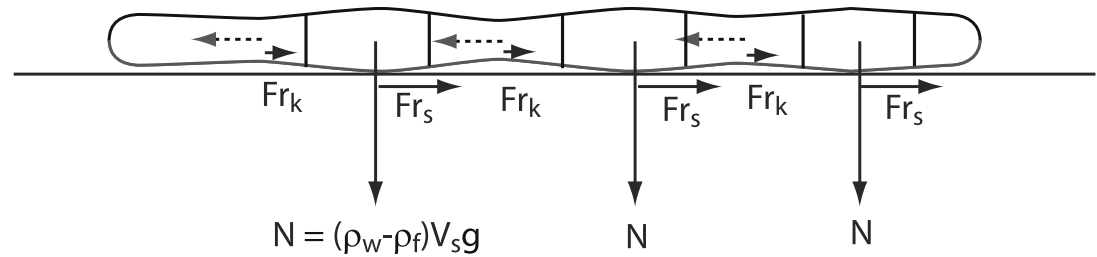

(b) Water

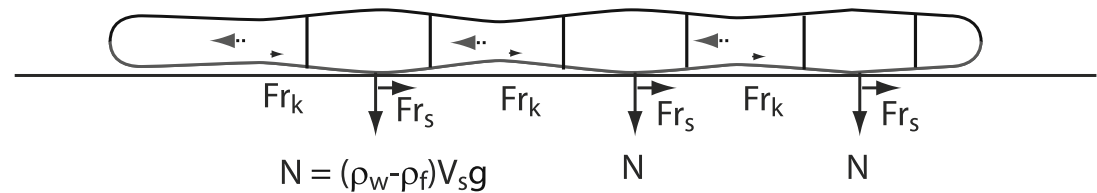

(c)

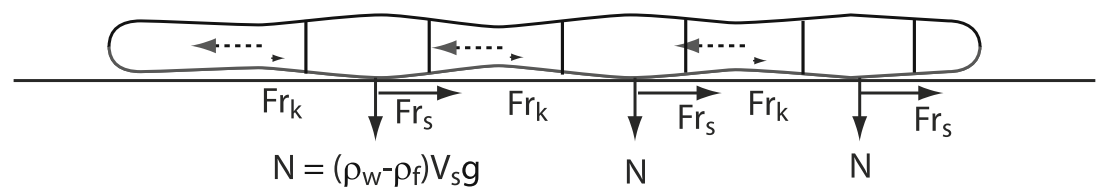

forward. Assuming that the weight of the worm is evenly distributed across all segments, we can solve for $q$ [1]:

$q<\frac{\mu_{\text {back }}}{\mu_{\text {back }}+\mu_{\text {forward }}}$

It follows that having mechanisms to increase $\mu_{\text {back }}$ relative to $\mu_{\text {forward }}$ should be advantageous in moving the most segments at a time and therefore moving at a greater absolute velocity. Earthworms have setae that point rearward, increasing the static friction coefficient, $\mu_{\text {back }}$ [27] and also increase friction by extending the setae further out of the body [28]. Nereidid polychaetes use parapodia with setae to generate thrust against the substratum; to move more rapidly, longitudinal muscle contraction is coordinated with parapodia to move by undulation [11].

The viscoelastic properties of the mucus used by gastropods enables them to resist backward sliding while reducing friction to move forward while crawling [29, 30]. The part of the foot exerting force to move forward applies small strains under which the mucus is a viscoelastic solid. Under large strains while the slug is moving, the yield stress is exceeded and the mucus behaves like a fluid. Moreover, the mucus recovers its elastic properties quickly after the slug stops, enabling it to push with the next muscular wave.

Another approach used by organisms to increase the static frictional force relative to forward kinetic friction is to increase the normal force, or the proportion of the weight distributed over the stationary segments, rather than increasing the friction coefficient. Contraction of circular muscles in the advancing segments of crawling earthworms decreases the diameter of moving segments [31], which likely results in a greater proportion of body mass being supported by the stationary segments. The moving segments are also often raised off of smooth surfaces [32], reducing kinetic friction. Gastropods may also reduce forward friction by focusing the body weight on the stationary parts of the foot. Lissmann [33] suggested that the parts of the foot that were moving were lifted up, and Jones and Trueman [34] showed that limpets have muscle fibers that seem adapted for lifting the foot. It is unlikely that the foot is lifted completely off the surface because the thin layer of mucus between the foot and the substratum makes it hard to physically raise the foot [13]. Muscular contractions may, however, shift part of the body weight off of the moving regions, reducing the kinetic friction without physically lifting the foot. If the moving segments are lifted, work against kinetic friction is less important and the number of moving segments, $q$, depends more on the proportion of the body needed to support the weight than on overcoming friction.

Earthworms crawling by peristalsis slip on smooth surfaces such as glass [32]. Gastropods slip less with their muscular foot because of adhesion from mucus, and they, as well as crawlers such as leeches and caterpillars that grasp or adhere to surfaces, are able to move on inclined surfaces much more effectively. The pro-legs of caterpillars grip the substratum, allowing the caterpillar to pull itself forward [35], essentially greatly increasing $\mu_{\text {back }}$ for the stationary segments. Caterpillars use surprisingly similar kinematics when climbing vertical surfaces as when 
crawling horizontally [36]. Even more extreme are leeches, which use two-anchor crawling and adhere with one end using suckers while moving the rest of the body forward either with the body close to the substratum in vermiform crawling or by taking long strides with the body raised higher above the substratum in inch-worm crawling [37]. Earthworms can use their peristomium to create suction and adhere to smooth surfaces to reduce slipping [32], but whether this behavior occurs in the natural environment and its importance to locomotion are unclear.

Due to buoyancy, underwater crawlers experience reduced effective weights compared to crawlers in air, and the difference in gravitational force affects locomotion directly in terms of the increased cost of maintaining body shape in air as well as indirectly through the static and kinetic frictional forces. This effect has been clearly demonstrated for crabs, which exhibit different walking gaits on land and under water [38]. For crawlers that move more slowly and lower in the bottom boundary layer than crabs, lift and drag forces are much less important, but the buoyancy difference remains. Energetic cost of transport is much higher for terrestrial slugs [13] than for abalone [17]. Although this difference is largely attributable to the cost of mucus production [13], the cost of maintaining body shape against gravitational forces may also play a role. Many terrestrial crawlers have structures such as the shells of snails and septa and outer cuticle of earthworms to help maintain body shape, but gravity does affect the pressure distribution within a hydrostat and likely increases the muscular work needed to move in air. Perhaps more importantly, frictional forces are directly proportional to the normal force, which is greatly reduced in a dense fluid [Fig. 1(b)]. For crawlers in water, work needed to overcome kinetic friction is much lower than in air, and mechanisms to increase the friction coefficient or to generate thrust by pushing backwards off of rough surfaces or gripping the substratum to exert force are likely more important [Fig. 1(c)].

Gravitational forces are important not only in distinguishing crawling in air from in water, but affect organisms that carry dense structures. The increased cost of carrying the shell doubles the cost of transport for terrestrial hermit crabs [39]. This added weight may be advantageous under water: extra weight directly increases the static friction and may allow the animal to exert greater thrust.

\section{Burrowing in Mud}

Muddy marine sediments are elastic solids in which bubbles grow [3] and worms extend burrows by fracture [5]. The burrow around the polychaete, Nereis virens, is a tongue-depressor-shaped crack that extends laterally away from the worm and compresses the worm dorsoventrally
(Fig. 2) [5]. Nereis everts its pharynx, extending the throat region anteriorly out of the mouth like a balloon, to exert a dorsoventral force on the walls of the crack, and stress in the sediment is amplified at the crack tip. The worm drives itself forward like a wedge, extending the crack anteriorly [40]. Forward movement during burrowing is undulatory, although visualization of stresses around the burrowing worm show stress patterns in gelatin resulting from an anterior-traveling peristaltic wave [40]. Quantitative kinematic comparisons to crawling or swimming nereids have not been conducted, but the constraints of lateral crack edges suggest that the amplitude of the undulatory wave is likely smaller for burrowing worms.

This mechanism of burrowing by crack propagation has been demonstrated in gelatin for several species of worms $[5,41]$, and is consistent with descriptions of morphologies and behaviors of a much broader range of burrowers; alternating expanded regions described in a diverse range of burrowers as part of a dual-anchor system of burrowing may serve a primary function of exerting stress against crack walls, only secondarily acting as anchors holding part of the body in place while another part moves forward [7, 24]. Clams are wedge-shaped and have been described as burrowing using the dual-anchor system, with the foot and expanded shell acting as alternating anchors [42]. The cirratulid, Cirriformia moorei, has recently been shown to use a similar wedge mechanism to extend its burrow but creates an anterior expansion with its hydrostatic skeleton rather than an eversible pharynx [41]. Although no studies have directly tested this mechanism for terrestrial burrowers, earthworms do exert larger radial than axial forces $[43,44]$, and these forces are highest near the anterior end [45], consistent with this mechanism of burrowing. Moreover, internal pressures of earthworms are not only higher while burrowing than crawling but also show different patterns corresponding to muscle contraction: pressure peaks for crawling earthworms correspond to contraction of circular muscles to elongate the segment and extend the body forward, whereas burrowing earthworms exhibit higher pressures during longitudinal muscle contraction and radial expansion of the body [45].

The major components of work needed to burrow through muddy sediments include the work to extend the crack and work to maintain the body shape against the elastic restoring force of the sediment [46]. Here, the term burrowing refers to moving through sediments by extending a burrow rather than moving within an already formed burrow or tube, which will be discussed below. The dimensionless wedge number $(\mathrm{Wg})$ is the ratio of the work of fracture to the elastic work,

$W g=\frac{G_{c}}{E h} \frac{w_{\text {crack }}}{w_{\text {worm }}}$, 

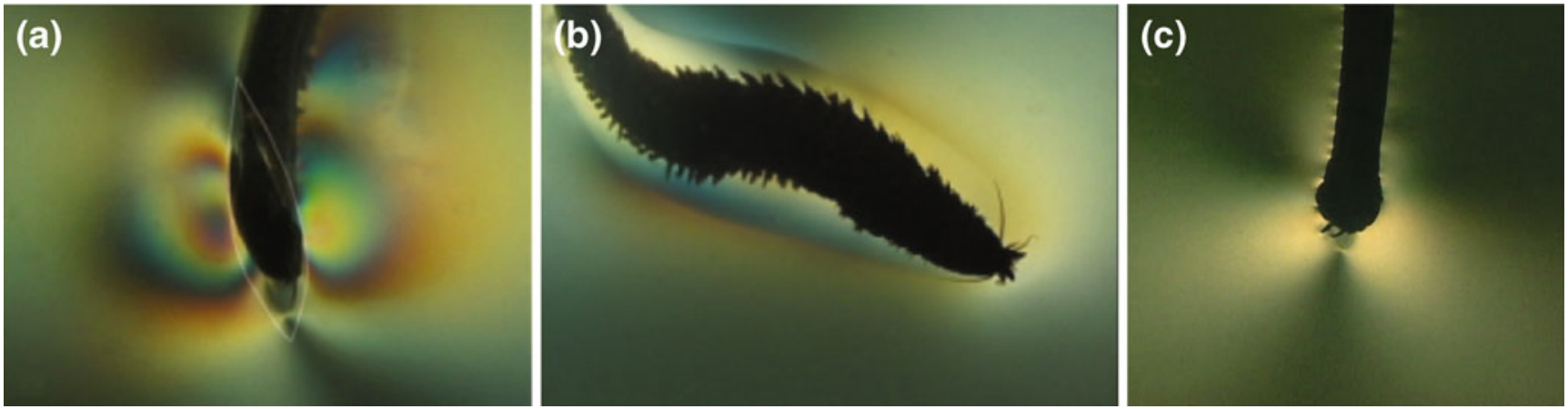

Fig. 2 The crack-shaped burrow around Nereis virens. (a), Anterior view in cross-polarized light, showing the longer axis of the discoidal crack oriented parallel with the setae in the crack and the dorsal and ventral surfaces against the gelatin. The stress field shown here results from displacement of the gelatin by the worm's body and reflects the elastic properties of the medium. The worm is not actively propagating the crack in this frame. (b), Dorsal view in cross-polarized light, showing the shape of the burrow around the animal and the low-force undulatory motion of the animal moving forward into the crack. (c), Side view in circularly polarized light, showing the stress field dorsal and ventral to the worm along the shorter axis of the discoidal crack and the crack extending anteriorly (from Dorgan et al. [5])

where $G_{c}$ is the fracture toughness $\left[\mathrm{J} \mathrm{m}^{-2}\right], E$ is the elastic modulus $[\mathrm{Pa}], h$ is the half-thickness of the worm [m], and $w_{\text {crack }}$ and $w_{\text {worm }}$ are the widths of the crack and the worm [m], respectively [46]. Nereis virens exhibits different behaviors in materials with different ratios of fracture toughness to stiffness $\left(G_{c} / E\right)$. In tough materials, $N$. virens everts its pharynx to become a thicker wedge and exert more force to extend the crack, and in stiffer materials it extends the crack farther laterally to reduce the elastic force compressing the body [46]. The wedge number is also used to predict the effect of body size on burrowing kinematics: smaller worms (with smaller $h$ ) have relatively thicker and blunter body shapes, consistent with the prediction that sediment is tougher to fracture for small worms [41].

Calculation of these work components assumes that sediment behaves linear elastically. On time scales of crack extension by fracture, this assumption provides a good approximation of reality [3], but the presence of permanent burrow structures in sediments indicates that this assumption is less accurate over the longer time periods that worms maintain body shape in sediments. The work against the elastic restoring force is calculated as the elastic stored energy, assuming none of that energy is utilized by the burrower to conserve energy. If burrowers could re-use some of this stored energy, the denominator term would be decreased by a factor corresponding to the conserved energy. Another potentially important consideration is whether the dissipation of stored energy results in passive permanent deformation or if the burrower does additional work to create a permanent burrow. The mechanism by which a crack becomes a cylindrical burrow is not known, but is an important area for future research. Permanent burrows are clearly present and abundant in sediments [47, 48], but whether burrowers can move through sediments without leaving a permanent trace is, quite literally, unclear. It is also important to note that both the elastic modulus, $E$, and fracture toughness, $G_{c}$, increase with depth in the sediment due to compaction, and that grain size (and therefore cohesion) has a strong effect on both properties, but more research on the depth-dependence of these properties is needed [49].

The static and kinetic frictional forces discussed for crawling on a surface are relevant for moving the body forward into the crack, but do not constitute the major components of work. The mechanics of burrowing have been described as a dual-anchor system, in which an expansion of the body anchors the animal in place while the anterior end extends forward into the substrate. The anterior end then expands to form a terminal anchor to hold the animal in place while the posterior end is pulled forward [24]. This description is consistent with the mechanism of crack propagation, with the 'anchors' reinterpreted to serving a primary function of exerting a force to extend the tip of the burrow by fracture [7]. A secondary function of these expansions is to serve as anchors, using static friction to keep the body from slipping backwards like the stationary segments of crawlers. The difference is that burrowers have two walls instead of one surface, and the normal force is primarily from the elasticity of the sediment although depending on the orientation of the worm, the weight of the overlying sediment may contribute as well [Fig. 3(a, b)]. These normal forces are much greater than gravitational forces on crawlers, especially under water. The static friction force must be large enough for the burrower to generate thrust to both overcome kinetic friction and to deform the sediment to make room for the anterior of the body as it moves forward. Static and kinetic frictional forces are smaller than normal forces, as indicated by friction coefficients, which are the ratios of frictional to normal forces under steady conditions, of less than one. Although the normal forces for burrowers are large, it seems likely that kinetic friction forces are 
(a)
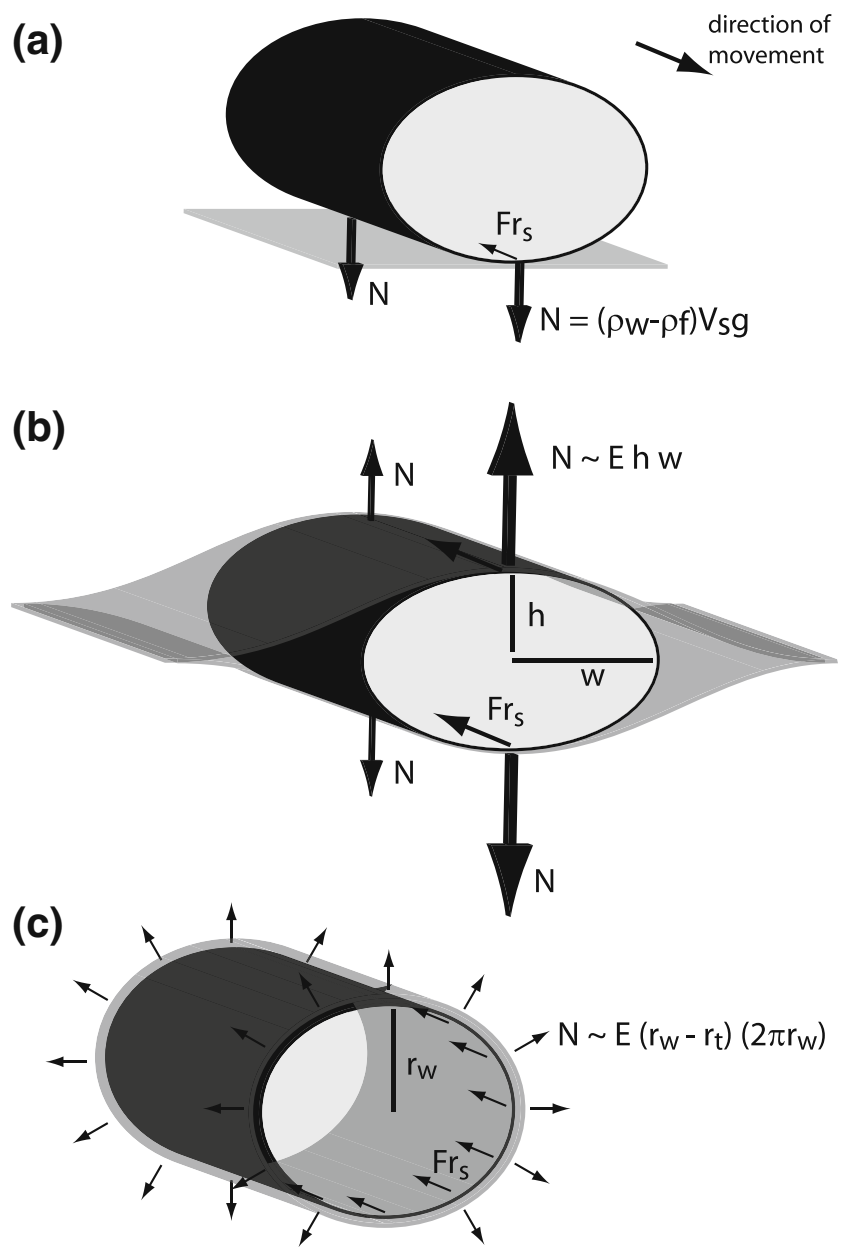

Fig. 3 Diagram of normal $(\mathrm{N})$ and frictional $\left(\mathrm{Fr}_{\mathrm{s}}\right)$ forces for a worm (a) crawling, (b) burrowing in mud, and (c) moving in tube. The normal force is the same as in Fig. 1 for the crawling worm, but for burrowing in mud is proportional to Ehw in which $E$ is the elastic modulus, $h$ is the half-thickness of the worm, and $w$ is the half-width of the worm, as indicated. For the worm in the tube, the normal force is proportional to $E\left(r_{w}-r_{t}\right)\left(2 \pi r_{w}\right)$ in which $r_{w}$ and $r_{t}$ are the radii of the worm and the tube respectively

smaller than those predicted from the kinetic friction coefficient because the animal is exerting force on the cohesive, elastic mud with the stationary part of the body while little force is exerted by the moving segments [40]. This is similar to mechanisms identified for crawlers to increase the static relative to kinetic friction, except burrowers have the added advantage of two surfaces against which to exert normal force rather than only one. In addition, burrowers using peristalsis extend a thin anterior region and then expand the body to push against the crack walls [41]. Separating anterior progression from thickening reduces the kinetic friction against which the worm must do work to move forward. It is important here to distinguish burrowing through muds, in which most of the force is applied perpendicular to the direction of movement and worms have little appreciable forward momentum, from friction-dominated processes such as driving a cone penetrometer into sediment, in which friction acts against a substantial driving force.

Consideration of the different components of work involved in burrowing and crawling suggests different strategies for the two types of locomotion (Fig. 3). Reducing kinetic friction by making the stationary segments thicker than the moving segments is likely easier for burrowers that push against two walls. Most of the work to burrow is done by the anterior region and involves extending the burrow and deforming the sediment in front of the worm to make room for the body. Burrowing polychaetes have muscular or expansible anterior regions [7], and when earthworms become desiccated, they maintain internal pressure in the anterior region [50]. For crawlers, reducing kinetic friction reduces the total work, suggesting advantage in reducing the contact area between the animal and the substratum, either by having a body with a lower surface area-to-volume ratio or by having bilateral appendages such as the pro-legs of caterpillars or large parapodia of nereid polychaetes. For burrowers, reducing the work to maintain body shape in the elastic sediment is likely important. This suggests an advantage in being thin, although the trade-offs are that enough force needs to be exerted to crack the sediment, requiring a thick enough wedge, and that most burrowers are deposit feeders that require large gut volumes to process sediments. One solution is to have a muscular anterior region with an eversible pharynx and a very long body (for gut volume), for example, that of capitellid polychaetes. If kinetic friction were a major component of external work, one would expect burrowing worms to have a lower surfacearea-to-volume ratio, or, essentially, to be less wormshaped.

\section{Burrowing in Sand}

Whereas the mechanics of muddy sediments are dominated by adhesive and cohesive forces binding the mucopolymeric matrix of organic material in which grains are suspended [3], sands comprise much larger grains that rest on each other under gravitational forces and transmit stresses at contact points [51]. Sands have lower organic content than muds, and when submerged their interstitial spaces contain water rather than the aqueous gels in finer sediments [52]. Because of irregular packing of grains, a small number of grains bear a disproportionate amount of the overlying weight, which is distributed along contact points that form stress arches and chains [51]. For burrowers close to the size of sand grains, this means that some grains are relatively easier to move than others. For burrowers much larger than the grain size, fluidization of 
sand involves disruption and rearrangement of stress chains $[6,53]$. In general, displaced grains fall back down under gravitational forces that differ in air from in water, where buoyancy and viscosity slow settling rates.

This suggests two different strategies for burrowing by macrofauna in sands: to fluidize sediments, disrupting stress chains and suspending grains, then moving quickly into the open space before the grains settle or, on a smaller scale, to move more slowly by breaking stress chains and removing or repacking grains [7]. The latter is likely important for small and/or slow moving burrowers. Unfortunately, few studies on soft-bodied sand-burrowers have been conducted, but some insight can be gained by considering strategies utilized by burrowers with external skeletons. For example, burrowing urchins move more slowly, have spines and tube feet to move individual grains, and are dome-shaped, which works well to withstand the weight of the overlying sand [54]. In contrast, urchins that burrow in mud are more wedge-shaped and rock up and down, potentially exerting forces on alternate walls of a crack-shaped burrow [7, 54]. The former strategy, fluidizing sands, is exemplified by the mole crab, Emerita, that burrows very quickly into sandy beaches but burrows only in saturated sands in which grains settle slowly [55]. Interestingly, sand lizards "swim" by undulation through desert sands at very fast speeds, up to $10 \mathrm{~cm} \mathrm{~s}^{-1}$ [6], compared to $1 \mathrm{~cm} \mathrm{~s}^{-1}$ for Emerita [55]. This velocity difference could be explained by the difference in buoyancy between air and water, although the sand lizards are much larger than Emerita, which may be a more important factor. In addition to buoyancy differences, unsaturated sands retain some water that holds grains together by capillary forces [56], making drained beach sands more difficult to burrow through than completely dry desert sands. The wave efficiency, the ratio of forward speed to wave speed, indicates how much the animal slips when moving by undulation, with low values of 0.25 for swimmers at low Reynolds numbers representing large slippage and high values closer to 1 for moving through a tube or crawling on a surface with minimal slippage [6]. Sandfish wave efficiencies fall in the middle, around 0.5, indicating intermediate slipping and transport of sand grains as the animal moves [6]. The drag on the sandfish, indicated by a wave efficiency much lower than one, likely comes from reorganization of stress chains and transport of particles when the sand is fluidized [6].

Shimada et al. [53] developed a simplified model of sand swimming by lizards as alternating expansion of two disks connected by a spring, similar to the dual anchor system described for burrowers [24]. A peak in velocity as a function of frequency of "burrowing cycle" indicates that an optimal velocity exists beyond which the sand is too fluidized and the "animal" is unable to get enough grip to push forward [53]. An optimal efficiency as a function of frequency was also observed, likely reflecting an intermediate level of fluidization such that the granular material acts as a solid around the "anchored" disk but is fluidized around the moving disk. This frequency is unsurprisingly lower than that for maximum velocity-it's possible to burrow faster but increased slipping and grain movement makes burrowing at higher velocities less efficient. This seems a likely mechanism for burrowing in sandy sediments, especially by larger infauna such as glycerid and nephtyid polychaetes that have morphologies and behaviors consistent with a dualanchor system. Both experiments on sand swimmers and numerical modeling, however, assume a much more ideal granular material than marine sands, which contain high concentrations of bacteria that produce exopolymers that glue particles together [57]. Fluidization is likely affected by those adhesive forces in natural sediments.

Clearly, more research is needed on burrowing with hydrostatic skeletons in sands to compare mechanisms in these different media. It seems, however, that the behaviors of burrowers in sands and muds are very similar, even though the mechanical responses of the sediments differ. For example, burrowers in both media move their heads from side to side [7]. Rather than serving to fluidize sediments [58], in muds this movement extends the crack edges laterally away from the worm, reducing the compressive force of the elastic material [46]. In sands, these head movements likely break stress chains and move grains into a tighter packing configuration. Burrowers in both media use alternating body expansions to move, but sands and muds respond differently to these forces, fluidizing and cracking, respectively.

\section{Moving in a Cylindrical Burrow or Tube}

Moving through a cylindrical burrow or tube requires the same balance of static and frictional forces as crawling on a surface, but the normal force is primarily elastic rather than gravitational and is obviously distributed cylindrically around the burrower rather than on one side [Fig. 3(c)]. Tube-dwellers need to move up and down in the tube and also need to resist being pulled out of the tube by predators. Many tube-dwellers have mechanisms such as hooked setae that work in one direction to grip the tube walls [59], which allows them to exert a large static frictional force without needing to overcome much kinetic friction. Moreover, these large static frictional forces can be exerted with minimal normal force because of the high friction coefficient. Worms therefore need to do very little work in the direction perpendicular to the axis of the tube compared to that needed to make a burrow. Tubedwellers do, however, need to do considerable work to build their tube or burrow structure. 


\section{Conclusions}

Although earthworms both crawl and burrow by peristalsis, nematodes by undulation, and gastropods using a muscular foot, the mechanics and energetics of locomotion depend on the physical environment, including whether the animal is in air or in water, on top of or within the substratum, and the mechanical properties of that substratum. Dominant components of external work to crawl are frictional or adhesive, whereas burrowers in muds do work to extend their burrow by fracture and to deform the elastic sediment. In sands, work to resuspend and repack grains against gravity is important, and for tube-dwellers, gravitational potential work is done to move upward, and frictional work is important in staying in the tube. The mechanics of locomotion depend on the physical environment and reflect different strategies to reduce the dominant external work terms. Comparisons of burrowing and crawling, or even burrowing in different substrata, should be done with careful consideration of the mechanical constraints of these different environments on animal locomotion.

Acknowledgments I thank PA Jumars and two anonymous reviewers for helpful comments on the manuscript, E Lauga and $\mathrm{P}$ Hosoi for organizing this issue, and funding from NSF IOS grant \#0642249 to MAR Koehl.

Open Access This article is distributed under the terms of the Creative Commons Attribution Noncommercial License which permits any noncommercial use, distribution, and reproduction in any medium, provided the original author(s) and source are credited.

\section{References}

1. Alexander RM (2003) Principles of animal locomotion. Princeton University Press, Princeton

2. Vogel S (1994) Life in moving fluids: the physical biology of flow. Princeton University Press, Princeton

3. Johnson BD, Boudreau BP, Gardiner BS, Maass R (2002) Mechanical response of sediments to bubble growth. Mar Geol 187:347-363

4. Duran J (2000) Sands, powders, and grains: an introduction to the physics of granular materials. Springer, New York

5. Dorgan KM, Jumars PA, Johnson BD, Boudreau BP, Landis E (2005) Burrow elongation by crack propagation. Nature 433:475

6. Maladen RD, Ding Y, Li C, Goldman D (2009) Undulatory swimming in sand: subsurface locomotion of the sandfish lizard. Science 325:314-318

7. Dorgan KM, Jumars PA, Johnson BJ, Boudreau BP (2006) Macrofaunal burrowing: the medium is the message. Oceanogr Mar Biol 44:85-121

8. Clark RB, Hermans CO (1976) Kinetics of swimming in some smooth-bodied polychaetes. J Zool Lond 178:147-159

9. Drewes CD, Fourtner CR (1993) Helical swimming in a freshwater oligochaete. Biol Bull 185:1-9

10. Clark RB, Tritton DJ (1970) Swimming mechanisms in nereidiform polychaetes. J Zool Lond 161:257-271
11. Gray J (1939) Studies in animal locomotion VIII. The kinetics of locomotion of Nereis diversicolor. J Exp Biol 16:9-16

12. Caldwell SL, Donovan DA (2003) Energetics of swimming and crawling in the lion nudibranch, Melibe leonina. Veliger 46:355-361

13. Denny M (1980) Locomotion: the cost of gastropod crawling. Science 208:1288-1290

14. Casey TM (1991) Energetics of caterpillar locomotion: biomechanical constraints of a hydraulic skeleton. Science 252:112-114

15. Berrigan D, Lighton JRB (1993) Bioenergetic and kinematic consequences of limblessness in larval dipteral. J Exp Biol 179:245-259

16. Walton M, Jayne BC, Bennett AF (1990) The energetic cost of limbless locomotion. Science 249:524-526

17. Donovan D, Baldwin J, Carefoot T (1999) The contribution of anaerobic energy to gastropod crawling and a re-estimation of minimum cost of transport in the abalone, Haliotis kamtschatkana (Jonas). J Exp Mar Biol Ecol 235:273-284

18. Trevor JH (1978) The dynamics and mechanical energy expenditure of the polychaetes Nephtys cirrosa, Nereis diversicolor, and Arenicola marina during burrowing. Estuar Coast Mar Sci 6:605619

19. Hunter RD, Elder HY (1989) Burrowing dynamics and energy cost of transport in the soft-bodied marine invertebrates Polyphysia crassa and Priapulus caudatus. J Zool 218:209-222

20. Schmidt-Nielsen K (1972) Energy cost of swimming, flying, and running. Science 177:222-228

21. McMahon TA, Greene PR (1979) The influence of track compliance on running. J Biomech 12:893-904

22. Zamparo P, Perini R, Orizio C, Sacher M, Ferretti G (1992) The energy cost of walking or running on sand. Eur J Appl Physiol 65:183-187

23. Kier WM, Smith KK (1985) Tongues, tentacles and trunks: the biomechanics of movement in muscular-hydrostats. Zool J Linn Soc 83:307-324

24. Clark RB (1964) Dynamics in Metazoan evolution. Oxford University Press, London

25. Koehl MAR, Quillin KJ, Pell CA (2000) Mechanical design of fiber-wound hydraulic skeletons: the stiffening and straightening of embryonic notochords. Am Zool 40:28-41

26. Elder HY (1980) Peristaltic mechanisms. In: Elder HY, Trueman ER (eds) Aspects of animal movement. Cambridge University Press, Cambridge, pp 71-92

27. Garrey WE, Moore AR (1915) Peristalsis and coordination in the earthworm. Am J Physiol 39:139-148

28. Murakami Y, Uchiyama H, Kurata J, Maeda M (2006) Dynamical locomotion analysis and a model for the peristaltic motion of earthworms. SICE-ICASE International Joint Conference Oct. 1821, 2006 in Bexco, Busan, Korea, 4224-4229

29. Denny M (1980) The role of gastropod pedal mucus in locomotion. Nature 285:160-161

30. Denny MW, Gosline JM (1980) The physical properties of the pedal mucus of the terrestrial slug, Ariolimax columbianus. J Exp Biol 88:375-393

31. Quillin KJ (1999) Kinematic scaling of locomotion by hydrostatic animals: ontogeny of peristaltic crawling by the earthworm Lumbricus terrestris. J Exp Biol 202:661-674

32. Yapp WB (1956) Locomotion of worms. Nature 177:614-615

33. Lissman HW (1945) The mechanism of locomotion in gastropod molluscs II. Kinetics. J Exp Biol 22:37-50

34. Jones HD, Trueman ER (1970) Locomotion of the limpet Patella vulgata L. J Exp Biol 52:201-216

35. Trimmer BA, Issberner J (2007) Kinematics of soft-bodied, legged locomotion in Manduca sexta larvae. Biol Bull 212:130-142

36. van Griethuijsen LI, Trimmer BA (2009) Kinematics of horizontal and vertical caterpillar crawling. J Exp Biol 212:1455-1462 
37. Stern-Tomlinson W, Nusbaum MP, Perez LE, Kristan WB Jr (1986) A kinematic study of crawling behavior in the leech, Hirudo medicinalis. J Comp Physiol A 158:593-603

38. Martinez MM, Full RJ, Koehl MAR (1998) Underwater punting by an intertidal crab: a novel gait revealed by the kinematics of pedestrian locomotion in air versus water. J Exp Biol 201:26092623

39. Herried CF, Full RJ (1986) Energetics of hermit crabs during locomotion: the cost of carrying a shell. J Exp Biol 120:297-308

40. Dorgan KM, Arwade SR, Jumars PA (2007) Burrowing in marine muds by crack propagation: kinematics and forces. J Exp Biol 210:4198-4212

41. Che J, Dorgan KM (2010) It's tough to be small: dependence of burrowing kinematics on body size. J Exp Biol 213:1241-1250

42. Trueman ER (1983) Locomotion in molluscs. In: Saleuddin ASM, Wilbur KM (eds) The mollusca. Academic, New York, pp 155198

43. Keudel M, Schrader S (1999) Axial and radial pressure exerted by earthworms of different ecological groups. Biol Fertil Soils 29:262-269

44. Quillin KJ (2000) Ontogenetic scaling of burrowing forces in the earthworm Lumbricus terrestris. J Exp Biol 203:2757-2770

45. Seymour MK (1969) Locomotion and coelomic pressure in Lumbricus terrestris L. J Exp Biol 51:47-58

46. Dorgan KM, Arwade SR, Jumars PA (2008) Worms as wedges: effects of sediment mechanics on burrowing behavior. J Mar Res 66:219-254

47. Shull DH, Yasuda M (2001) Size-selective downward particle transport by cirratulid polychaetes. J Mar Res 59:453-473

48. Dufour SC, Desrosiers G, Long B, Lajeunesse P, Gagnoud M, Labrie J, Archambault P, Stora G (2005) A new method for three- dimensional visualization and quantification of biogenic structures in aquatic sediments using axial tomodensitometry. Limnol Oceanogr: Methods 3:372-380

49. Johnson BD et al (submitted) Profiling fracture toughness in cohesive sediments. Geotechnique

50. Stovold RJ, Whalley WR, Harris PJ (2003) Dehydration does not affect the radial pressures produced by the earthworm Aporrectodea caliginosa. Biol Fertil Soils 37:23-28

51. Geng J, Howell D, Longhi E, Behringer RP, Reydellet G, Vannel L, Clement E, Luding S (2001) Footprints in sand: the response of a granular material to local perturbations. Phys Rev Lett 87:035506

52. Watling L (1988) Small-scale features of marine sediments and their importance to the study of deposit feeding. Mar Ecol Prog Ser 47:135-144

53. Shimada T, Kadau K, Shinbrot T, Herrmann HJ (2009) Swimming in granular media. Physical Review E 020301(R): 4 pp

54. Kanazawa K (1992) Adaptation of test shape for burrowing and locomotion in spatangoid echinoids. Palaeontology 35:733-750

55. Trueman ER (1970) The mechanism of burrowing of the mole crab, Emerita. J Exp Biol 53:701-710

56. Denny MW (1993) Air and water: the biology and physics of life's media. Princeton University Press, Princeton

57. Decho AW (1990) Microbial exopolymer secretions in ocean environments: their role(s) in food webs and marine processes. Oceanogr Mar Biol Annu Rev 28:73-153

58. Hunter RD, Moss VA, Elder HY (1983) Image analysis of the burrowing mechanisms of Polyphysia crassa (Annelida: Polychaeta) and Priapulus caudatus (Priapulida). J Zool 199:305323

59. Merz RA, Woodin SA (2000) Hooked setae: tests of the anchor hypothesis. Invertebr Biol 119:67-82 